\title{
Trial of Fabrication of Multiple-Tone Magneto-Optical Spatial Light Modulator for Analog Data Processing
}

\author{
A. Tsuzuki ${ }^{*}$, H. Takagi ${ }^{*, * *}$, P. B. Lim ${ }^{*, * * *}$, H. Uchida ${ }^{*}$, K. H. Shin ${ }^{*, * * * * *}$ and M. Inoue, $e^{*, * * *}$ \\ "Toyohashi University of Technology, 1-1 Hibari-Ga-Oka, Tempaku, Toyohashi, Aichi 441-8580, Japan \\ ** Toyota National College of Technology, 2-1 Eisei, Toyota, Aichi 471-8525, Japan \\ *** JST-CREST, 4-1-8 Honcho, Kawaguchi, Saitama 332-0012, Japan \\ ${ }^{* * * *}$ Kyungsung University, Daeyeon-dong, Nam-gu, Pusan 608-736, Korea
}

A magneto optic spatial light modulator (MO-SLM) for multiple-tone expression was fabricated by using in-plane magnetization of garnet film. By using Al substituted Bi:YIG film, magnetization curve was modified to suitable for multiple tone expression. Changing a driving current from 0 to $28 \mathrm{~mA}$, we were able to change brightness of pixels in the line.

Key words: Magneto-optic spatial light modulator, multiple torn, garnet, magneto-optical effect, current-driving

\section{Introduction}

A spatial light modulator (SLM) is one of prospective optical devices for optical communication technology, display technology and so on. The SLM can treat large amount of data as a page with two-dimensional pixel array. Although a liquid crystal SLM is a representative device, it has disadvantage of slow switching speed. In order to improve the switching speed, we had studied a magneto-optic spatial light modulator (MO-SLM)1)-4) as shown in Fig. 1 (a). A structure of the MO-SLM had a magneto-optical layer of garnet for pixels, and electrode to flow a current to produce a magnetic field, which utilizes magneto optical Faraday effect to modulate plane of polarized light in the garnet layer by direction of magnetization. These MO-SLMs had a garnet film with out-of-plane magnetic anisotropy, so magnetization of the garnet film was controlled in the out-of-plane direction (Fig. 1 (b)). Therefore, the MO-SLMs that we reported before can modulate light as binary information.

The Faraday rotation angle is given equation by

$$
\left.\theta_{F}=F\left(M / M_{S}\right)\right]
$$

where $\theta_{F}$ is Faraday rotation angle, $F$ is coefficient of Faraday rotation, $M$ is magnetization, $M_{S}$ is saturation magnetization, and $l$ is length of magneto-optical film. Intensity $I_{\text {out }}$ of the transmitted light from the garnet film is described by

$$
I_{\text {out }}=I_{\text {in }} * \cos ^{2} \theta
$$

where $\theta$ is angular differences of the plane of polarized light to a polarizer, $I_{\text {in }}$ is intensity of the input light.

In the previous MO-SLM (Fig. 1 (b)), the magneto-optical $\mathrm{Bi}$ substituted Yttrium Iron Garnet (Bi:YIG) layer was formed by liquid phase epitaxial (LPE) method, which the Bi:YIG film had easy axis of out-of-plane magnetization. Therefore, magnetization of magneto-optical Bi:YIG layer switched in vertical direction to the film, which was the same direction of transmitted light. So, the previous MO-SLM switched light as binary contrast by changing magnetization. However, it is not suitable to express an intermediate value.

In this study we tried to fabricate a new MO-SLM with multiple-tone expression, which can treat analog information with high speed. The multiple-tone MO-SLM (Fig. 1 (c)) has in-plane magnetization garnet film. Using polycrystalline garnet film as magneto-optical layer, direction of magnetization can change gradually in vertical direction for changing the intensity of light. The multiple-tone MO-SLM will have great influence in the field of optical information technology, such as light computing, projector and holographic recording.

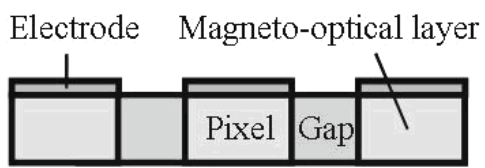

(a)

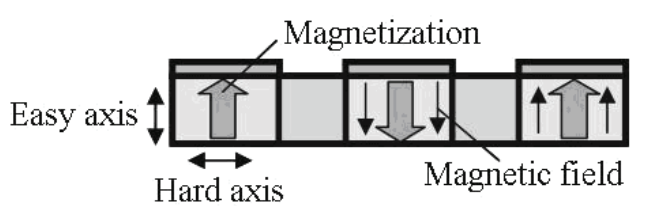

(b)

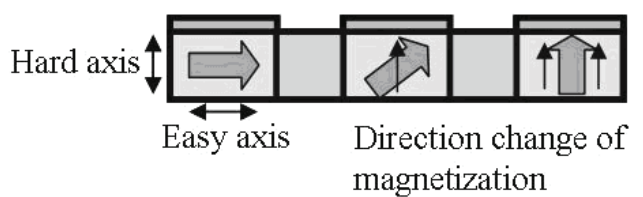

(c)

Fig. 1 Mechanism of switching of magnetization in magneto-optical garnet pixels of a MO-SLM. (a) Cross-section of basic structure, (b) traditional MO-SLM using out-of-plane magnetization, and (c) multiple-tone MO-SLM using in-plane magnetization. 


\section{Fabrication of the multiple-tone MO-SLM}

We fabricated the multiple-tone MO-SLM using in-plane magnetization by following processes. First, we prepared the amorphous garnet film with a thickness of $1 \mu \mathrm{m}$ on a Corning \#1737 glass. In order to achieve the multiple-tone expression, we used polycrystalline garnet with in-plane magnetization fabricated by $\mathrm{RF}$ magnetron sputtering method. Therefore, we used $\mathrm{Al}$ substituted $\mathrm{Bi}$ :YIG film (Bi,Al:YIG) by using $\mathrm{Al}_{2} \mathrm{O}_{3}$ pellets on a $\mathrm{Bi}_{1.0} \mathrm{Y}_{2.5} \mathrm{Fe}_{5} \mathrm{O}$ x target in order to modify magnetic property. Sputtering pressure of Ar gas was 3 mTorr, a substrate temperature was 100 degrees C. After deposition of the amorphous $\mathrm{Bi}, \mathrm{Al}$ :YIG film, it was crystallized by annealing in air. Figure 2 shows magnetic properties of the magneto-optical Bi,Al:YIG layer. By substitution of $\mathrm{Al}^{3+}$ ions for $\mathrm{Fe}^{3+}$ ions in crystallographic YIG structure, the saturation magnetization was decreased. The Bi,Al:YIG film with low saturation magnetization has advantage of operation of the multiple-tone MO-SLM that controls the Faraday rotation by an applied magnetic field using a current. The device was made by using the composition $(\mathrm{Al} / \mathrm{Fe}=0.055)$ that coercivity and saturation magnetization of in-plane are small.

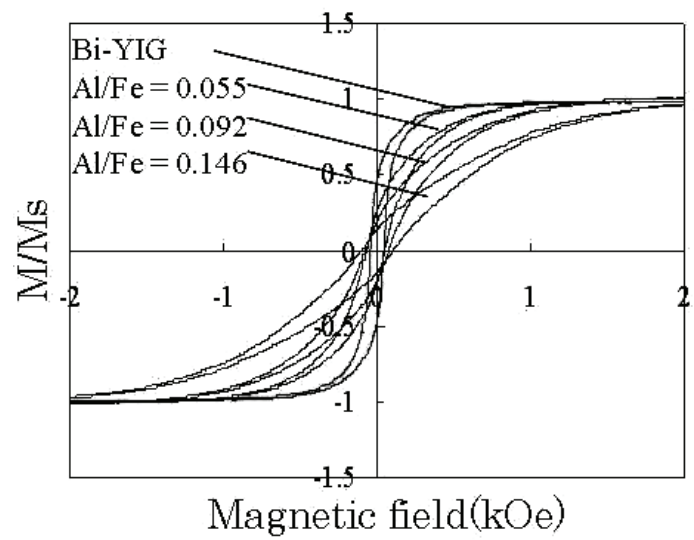

(a)

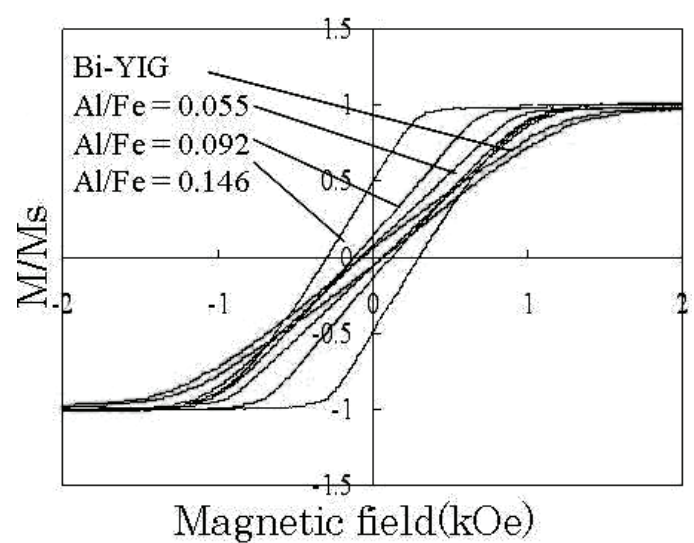

(b)

Fig. 2 Normalized saturation magnetization. (a) In-plane magnetization, (b) out-of-plane magnetization.
In following fabrication process of the MO-SLM, a photoresist mask for pixels was formed by photolithography on the Bi,Al:YIG film; the film was etched by phosphoric acid to separate pixels because of magnetic isolation. After the photoresist masks were removed by acetone, the $\mathrm{Bi}, \mathrm{Al}$ :YIG pixels were annealed for crystallization at 750 degrees $\mathrm{C}$ for 15 minutes in the air in an electric furnace. After the photoresist was deposited on the Bi,Al:YIG pixels and grooves between pixels by a spin coater, the extra photoresist was removed by polishing on the pixels. To prevent detachment of the photoresist inside grooves, $\mathrm{SiO}_{2}$ film with thickness of $400 \mathrm{~nm}$ was deposited by $\mathrm{RF}$ magnetron sputtering method as a protection layer. Then, an $\mathrm{Al}$ film for electrode was deposited on the $\mathrm{SiO}_{2}$ film by sputtering method; finally $\mathrm{Al}$ electrodes were formed by the photolithography.

\section{Evaluation of the multiple-tone MO-SLM}

Figure 3 shows change of brightness in pixels as a function of external magnetic field, which was obtained by a polarization microscope with a charge coupled device (CCD) camera. The brightness increased in proportion to the external magnetic field, whose inclination was about 30 a.u./kOe between -1.2 to 1.2 $\mathrm{kOe}$.

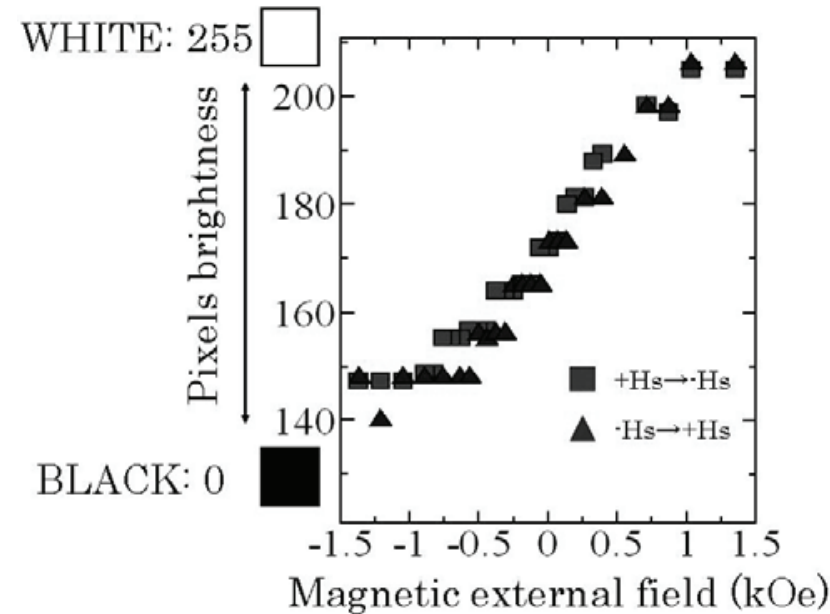

Fig. 3 Change of Brightness of pixels in the multiple-tone MO-SLM measured by a polarization microscope with a CCD camera. In the camera image, maximum was 255 A.U. and minimum was 0 A.U. in brightness

Figure 4 shows images of polarization microscope of the fabricated multiple-tone MO-SLM. Arrows indicates a line of pixels with an electrode flowing a driving current. When a driving current was changed from 0 to $28 \mathrm{~mA}$, brightness of magneto-optical images changed as seen from Fig. 4 (a) to (d). Therefore, we confirmed that the fabricated MO-SLM was able to control light in selected pixels by changing the magnetization from in-plane direction to out-of-plane direction.

Figure 5 shows change of brightness in pixels by the driving current, which was obtained by the 
polarization microscope with CCD camera. By an induced magnetic field by the driving current, the brightness of pixels was able to be controlled in the MO-SLM. However, in the experiments, the electrode of the MO-SLM disconnected by a current above $28 \mathrm{~mA}$. So, it must be solved this heating problem by decrease of electric resistance of the electrode, by design of electrode pattern for effective magnetic field, an area of cross-section of electrode and so on.

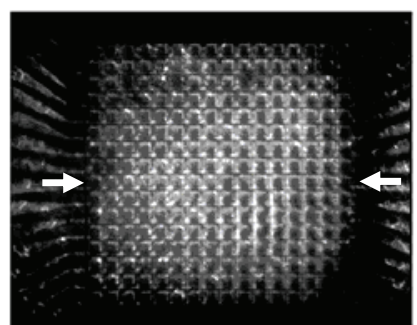

(a) $0 \mathrm{~mA}$

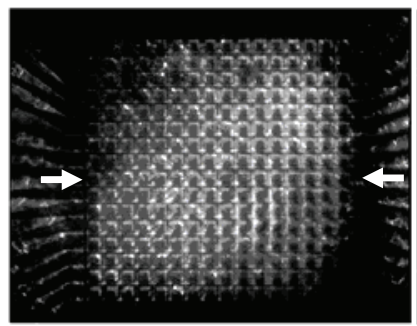

(b) $12 \mathrm{~mA}$

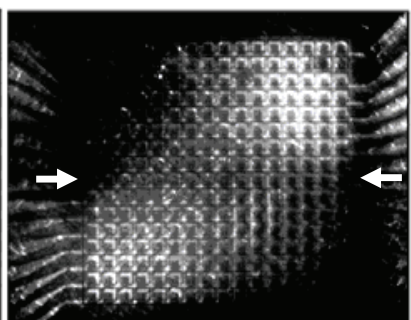

(c) $22 \mathrm{~mA}$

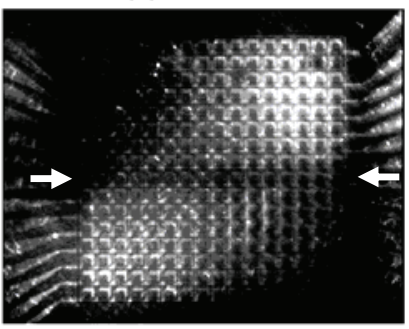

(d) $28 \mathrm{~mA}$
Fig. 4 Images of polarization microscope for magneto-optical evaluation of the multiple-tone MO-SLM. Arrows indicates a line flowing a driving current.

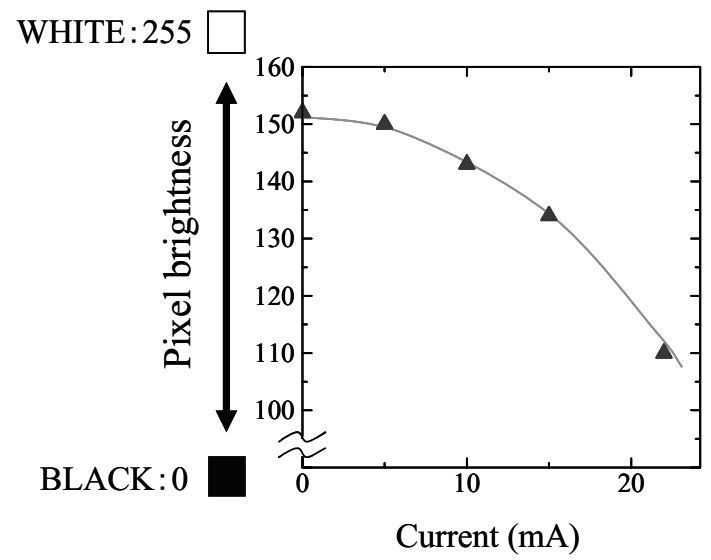

Fig. 5 Change of brightness of pixels as a function of a driving current, which was measured by the polarization microscope with CCD camera

\section{Conclusion}

For analog data processing, we tried to fabricate the multiple-tone MO-SLM using in-plane magnetization garnet film. To have in-plane magnetization, we used $\mathrm{Bi}, \mathrm{Al}$ :YIG film fabricated by $\mathrm{RF}$ magnetron sputtering method. By using the polarization microscope, change of brightness in pixels of the MO-SLM was measured when the driving current flowed from $0 \mathrm{~mA}$ to $28 \mathrm{~mA}$. In order to improve brightness for multiple-tone expression, optimization of device structure and magneto-optical layer will be required.

Acknowledgements The work was supported in part by the Super Optical Information Memory Project by MEXT and NEDO Development of Strategic Technologies of the Next Generation for Their Practical Uses.

\section{References}

1) J. H. Park, D. H. Lee, J. K. Cho and M. Inoue, J. Appl. Phys., 91, 7014 (2002).

2) J. H. Park, J. Cho, K. Nishimura and M. Inoue, Jpn. J. Appl. Phys., 41, 4B, 2541 (2002).

3) J. H. Park, J. K. Cho, and M. Inoue, Jpn. J. Appl. Phys., 41, 3B, 1813(2002).

4) H.Takagi, M.Mizoguchi, J. H. Park, K. Nishimura, H. Uchida, and M. Inoue, Proc. MRS, 785, D6 10.1(2004).

Received May 17, 2006; Received July 11, 2006;

Accepted Aug. 10, 2006 